\title{
The Haldane Energy Gap of A Doped Linear-Chain Heisenberg Antiferromagnet
}

\author{
Zhong-Yi Lu, ${ }^{a} \quad$ Zhao-Bin $\mathrm{Su},{ }^{a} \quad \mathrm{Lu} \mathrm{Yu}{ }^{a, b}$ \\ ${ }^{a}$ Institute of Theoretical Physics, Academia Sinica, \\ P. O. Box 2735, Beijing 100080, China \\ ${ }^{b}$ International Centre for Theoretical Physics, 34100 Trieste, Italy
}

\begin{abstract}
Using the valence-bond-solid (VBS) approach and the Schwinger boson mean field approximation, we study the dependence of the Haldane gap of a spin-1 linear chain Heisenberg antiferromagnet on impurity doping with different spins. The impurity spins affect the singlet pairing order parameter $\Delta$ and the constraint factor $\lambda$. As a result, the Haldane gap is reduced by a factor $\sim n_{i}^{2 / 3}$, with $n_{i}$ as the impurity concentration, and eventually collapses at $n_{i} \sim 1 / \xi$ with $\xi$ as the VBS correlation length. This theoretical prediction can be verified by neutron scattering experiments.
\end{abstract}

PACS numbers: 75.30.Ds, $75.30 . \mathrm{Hx}, 75.50 . \mathrm{Ee}$ 
Some years ago Haldane [1] conjectured that the excitation spectrum of a linear-chain Heisenberg antiferromagnet (LCHA) with integer spin has a finite energy gap $E_{H}$ above its singlet ground state, while a LCHA with half-integer spin has a gapless spectrum. This conjecture has been strongly supported by an exact solution of a specific model [2], numerical studies [3], [4] and experiments [5], [6]. Recently, there has been a revived interest in this problem due to the experimental observation of spin 1/2 degrees of freedom [7], [8] at the ends of a finite spin-1 chain induced by doping with different spins, as predicted by theory [2], [9].

P.W. Anderson [10] has proposed the Resonant-Valence-Bond (RVB) model to describe some spin 1/2 antiferromagnet systems. Affleck, Kennedy, Lieb and Tasaki (AKLT) [2] have generalized this idea to spin-1 LCHA by proposing the Valence-Bond-Solid (VBS) state. In this VBS state, the valence bonds formed by two $1 / 2$ spins as a singlet $\uparrow \downarrow-\downarrow \uparrow$, connect two nearest neighbors, while the two $1 / 2$ spins on the same site should be symmetrized to form a triplet state $S=1$. This is a translationally invariant, singlet state. The spin correlation functions decay exponentially and there is a gap $E_{H}$ in the energy spectrum which is closely related to the VBS order parameter.

Consider a generalized Heisenberg Hamiltonian

$$
H_{0}=J_{0} \sum_{i}\left[\vec{S}_{i} \cdot \vec{S}_{i+1}-\beta\left(\vec{S}_{i} \cdot \vec{S}_{i+1}\right)^{2}\right]
$$

where $-1 \leq \beta \leq 1$ is a parameter. The VBS state proposed by AKLT is an exact, nondegenerate ground state of (1) for $\beta=-1 / 3$. The theoretical analysis using the nonAbelian bosonization technique and the conformal field theory [11], as well as the exact diagonalization for small clusters [9] seems to show that systems described by (11) with $-1<\beta<1$ belong to the same universality class as the $\beta=-1 / 3$ case, so its properties are generic to all systems of this class. Recently, Ng [12] has applied the Schwinger boson mean field approximation (SBMFA) adopted by Arovas and Auerbach [13 for quantum spin 
discussed in his paper and will not be repeated here.

In this Letter we use the SBMFA to consider the effect of random doping with different spins on the Haldane gap. In this approach the Haldane gap $E_{H}$ is determined by the VBS order parameter $\Delta$ and the single-occupancy constraint factor $\lambda$. Upon doping, apart from the end states, $\Delta$ and $\lambda$ are modified by both spin-flip scattering and the difference in the spin values. By solving self-consistently the system of equations for the order parameter and the constraint we find that the reduction of the Haldane gap $E_{H}$ originating from the VBS structure is proportional to $n_{i}^{2 / 3}$, where $n_{i}$ is the impurity concentration, and it collapses when $n_{i}$ is comparable to $\xi^{-1}$, the inverse of the VBS correlation length. The situation here is rather similar to superconductors doped with paramagnetic impurities which break the time-reversal symmetry of the singlet pairing, giving rise to reduction and eventual collapsing of the energy gap [14]. Of course, if the spin chain is strictly one-dimensional, doping by non-magmetic impurities will break the chain. However, the real system is only quasi-onedimensional, so the interchain coupling as well as the superexchan The dynamic structure factor $S(q, \omega)$, measured in the neutron scattering experiments, is the Fourier transform of the spin-spin correlation function $<\vec{S}_{i}(t) \cdot \vec{S}_{j}(0)>$, where $<\cdots>$ means thermal average. Apart $\mathrm{f}$

The Hamiltonian of a LCHA doped with impurity spins can be written as [16]:

$$
\begin{gathered}
H=H_{0}+H^{i m}, \quad H_{0}=J_{0} \sum_{i} \vec{S}_{i} \cdot \vec{S}_{i+1}, \quad H^{i m}=\sum_{<i \alpha>} \vec{A}_{\alpha} \cdot \vec{S}_{i}, \\
\vec{A}_{\alpha}=g \vec{S}_{\alpha}^{i m}-J_{0} \vec{S}_{\alpha}, \quad \vec{S}^{2}=S(S+1),
\end{gathered}
$$

where $\vec{S}^{i m}$ is the impurity spin, $g$ is the coupling between impurity and host spins, $\alpha$ denotes impurity site, $\langle i \alpha>$ means summation over all impurity spins with $i=\alpha \pm 1$.

We will discuss a generic case when impurities are randomly distributed along the LCHA and the averages are $\overline{\vec{S}^{i m}}=0, \overline{\vec{S}_{\theta}^{2} i m}=1 / 3 S^{i m}\left(S^{i m}+1\right)($ where $\theta$ means any direction), $\overline{\overrightarrow{A^{2}}}=g^{2} S^{i m}\left(S^{i m}+1\right)+J_{0}^{2} S(S+1)$. 
$\left[a, b^{\dagger}\right]=0,\left[a, a^{\dagger}\right]=1$. It is convenient to use Nambu's four-component formalism to treat the Hamiltonian (2). Let $\psi_{i}=\left(a_{i}, b_{i}, b_{i}^{\dagger}, a_{i}^{\dagger}\right)^{T}, \psi(k)=\left(a_{k}, b_{k}, b_{-k}^{\dagger}, a_{-k}^{\dagger}\right)^{T}$, then $\psi_{i}^{\dagger}=$ $\left(a_{i}^{\dagger}, b_{i}^{\dagger}, b_{i}, a_{i}\right), \psi^{\dagger}(k)=\left(a_{k}^{\dagger}, b_{k}^{\dagger}, b_{-k}, a_{-k}\right)$, where $T$ means transposition and $k$ is the wave vector. To carry out the decoupling in the SBMFA we introduce the VBS order parameter $\Delta_{i j}=<a_{i} b_{j}-b_{i} a_{j}>$, with $i j$ denoting the nearest neighbor pair of sites.

We first consider the undoped system. In the SBMFA the Hamiltonian can be written as

$$
\begin{aligned}
H_{0} & =\sum_{i} \psi_{i}^{\dagger} M_{i, i+1} \psi_{i, i+1}+\sum_{i} \psi_{i}^{\dagger} \Lambda_{i} \psi_{i} \\
& +\sum_{i} J\left|\Delta_{0} i, i+1\right|^{2}+\sum_{i} \lambda_{0 i}(-1-2 S),
\end{aligned}
$$

where

$$
M_{i, i+1}=J\left(\begin{array}{cc}
0 & -\Delta_{0} i, i+1 \\
\Delta_{0 i, i+1}^{*} \sigma_{3} & 0
\end{array}\right), \quad \Lambda_{i}=\frac{\lambda_{0 i}}{2}\left(\begin{array}{ll}
I & 0 \\
0 & I
\end{array}\right),
$$

while $*$ means complex-conjugate, $J=J_{0} / 2, I$ and $\sigma_{3}$ are $2 \times 2$ unit and Pauli matrix, respectively.

Performing the gauge transformation of Read and Newns [17] to absorb the phase factor and to remove the redundancy of constraint, the Lagrangian corresponding to eq.(33) can be written in the momentum space as

$$
L=1 / 2 \sum_{k} \psi^{\dagger}(k)\left(\omega \Omega_{1}-\lambda_{0} \Omega_{2}+J \Delta_{0} \nu_{k} \Omega_{3}\right) \psi(k)+\text { const },
$$

where $\nu_{k}=2 \sin k, \Delta_{0}$ is the module of the VBS order parameter. Here we take the lattice spacing $a$ as the length unit and denote $\vec{\Omega}=\left(\Omega_{1}, \Omega_{2}, \Omega_{3}\right)$ as

$$
\Omega_{1}=\left(\begin{array}{cc}
I & 0 \\
0 & -I
\end{array}\right), \Omega_{2}=\left(\begin{array}{cc}
I & 0 \\
0 & I
\end{array}\right), \Omega_{3}=\left(\begin{array}{cc}
0 & \sigma_{3} \\
\sigma_{3} & 0
\end{array}\right) .
$$

From eq.(4) one obtains the boson Green function

$$
\omega \Omega_{1}+\lambda_{0} \Omega_{2}+J \Delta_{0} \nu_{k} \Omega_{3}
$$


where $\eta$ is a positive infinitesimal. The Schwinger boson excitations form a continuum band and their dispersion relation is $\epsilon(k)=\left(\lambda_{0}^{2}-4 J^{2} \Delta_{0}^{2} \sin ^{2} k\right)^{1 / 2}$.

Now we can easily calculate the dynamic structure factor at $T=0 K$ as follows

$$
\begin{aligned}
& S(q, \omega)=12 \pi \int \frac{d k_{1}}{2 \pi} \int \frac{d \omega_{1}}{2 \pi} \int \frac{d \omega_{2}}{2 \pi} \delta\left(\omega-\omega_{1}-\omega_{2}\right) \\
& \left\{\operatorname{Im} G_{044}\left(k_{1}, \omega_{1}\right) \operatorname{Im} G_{011}\left(q+k_{1}, \omega_{2}\right)-\operatorname{Im} G_{042}\left(-k_{1}, \omega_{1}\right) \operatorname{Im} G_{013}\left(q+k_{1}, \omega_{2}\right)\right\}
\end{aligned}
$$

where $G_{044}, G_{011}, G_{042}$ and $G_{013}$ are elements of the Green function matrix (5). Integrating over $\omega_{1}$ and $\omega_{2}$ of eq.(6), we find that the $\delta$-function representing the energy conservation becomes $\delta\left(\omega-\left(\lambda_{0}^{2}-4 J^{2} \Delta_{0}^{2} \sin ^{2} k_{1}\right)^{1 / 2}-\left(\lambda_{0}^{2}-4 J^{2} \Delta_{0}^{2} \sin ^{2}\left(q+k_{1}\right)\right)^{1 / 2}\right)$. Hence only when $\omega \geq 2\left(\lambda_{0}^{2}-4 J^{2} \Delta_{0}^{2}\right)^{1 / 2}$, a neutron can be scattered and there must be two Schwinger bosons to form a physical magnetic excitation for spin $S=1$. It is also clear from the SchwingerBoson constraint for the spin-1 chain $\left(a^{\dagger} a+b^{\dagger} b=2\right)$ that they must appear in pairs. This is entirely consistent with the description based on field-theoretical mappings [19]. Thus the Haldane gap $E_{H_{0}}$ is the bottom of the spin excitation continuum and is given by $E_{H_{0}}=2\left(\lambda_{0}^{2}-4 J^{2} \Delta_{0}^{2}\right)^{1 / 2}$. The parameters $\Delta_{0}$ and $\lambda_{0}$ can be consistently evaluated [12], [18]. At temperature $T=0 K$, they are $\lambda_{0}=4.7296 J, \Delta_{0}=2.36312, E_{H_{0}}=0.1772 J_{0}$.

Now we consider the doped system. In the following $\vec{A}_{\alpha}$ is referred to as a quasiclassical quantity. In the Nambu representation the impurity part of the Hamiltonian (2) can be written as $H^{i m}=1 / 4 \sum_{<i \alpha>} \psi_{i}^{\dagger}\left(\vec{A}_{\alpha} \cdot \vec{\Sigma}\right) \psi_{i}$, in which $\vec{\Sigma}=\left(\Sigma^{1}, \Sigma^{2}, \Sigma^{3}\right)$ and

$$
\Sigma^{1}=\left(\begin{array}{cc}
\sigma_{1} & 0 \\
0 & \sigma_{1}
\end{array}\right), \Sigma^{2}=\left(\begin{array}{cc}
\sigma_{2} & 0 \\
0 & \sigma_{2}
\end{array}\right), \Sigma^{3}=\left(\begin{array}{cc}
\sigma_{3} & 0 \\
0 & -\sigma_{3}
\end{array}\right),
$$

with $\sigma_{1}, \sigma_{2}, \sigma_{3}$ as Pauli matrices.

Since the impurities are randomly distributed, their average effect is zero if each impurity scatters a Schwinger boson only once. Thus the multiple-scattering effect should be considered. Analysing the Feynman graphs, one finds that the contributions from the crossing dia- 
function $\bar{G}(k, \omega)$ of the doped system after averaging over the random distribution recovers the translational invariance. Hence we have $\bar{G}^{-1}(k, \omega)=\bar{G}_{0}^{-1}(k, \omega)-\Sigma(k, \omega)$, where $\Sigma(k, \omega)$ is the self-energy term due to the impurity scattering and $\bar{G}_{0}^{-1}(k, \omega)=\omega \Omega_{1}-\lambda \Omega_{2}+J \Delta \nu_{k} \Omega_{3}$, which has the same form as eq.(5). However, parameters $\lambda$ and $\Delta$ should be self-consistently determined like $\lambda_{0}$ and $\Delta_{0}$ and in principle they are different from the latter due to the impurity effect. Furthermore, we make an ansatz that the inverse of the Green function $\bar{G}^{-1}(k, z)=\bar{Z} z \Omega_{1}-\bar{\lambda} \Omega_{2}+J \bar{\Delta} \bar{Z} \nu_{k} \Omega_{3}$, where $z$ is the frequency in the complex plane, $\bar{Z}$ is the wave function renormalization factor, $\bar{\lambda}$ and $\bar{\Delta}$ are the renormalized parameters for the $\lambda$-multiplier and the order parameter, respectively. All of them are functions of $k$ and $z$. The self-energy part after averaging over random distribution can be written as

$$
\Sigma(k, \omega)=n_{i} \int(\vec{A} \cdot \vec{\Sigma})^{2} \bar{G}\left(k_{1}, \omega\right) \cos ^{2}\left(k-k_{1}\right) \frac{d k_{1}}{2 \pi} .
$$

From the definitions given above and eq.(7), we obtain a closed set of integral equations to determine $\bar{Z}, \bar{\lambda}$, and $\bar{\Delta}$. Since the doping density $n_{i}$ is rather small, they can be expanded in powers of $n_{i}$ and we will keep only the leading order of $n_{i}$ in the following. Accordingly we obtain that 18

$$
\bar{Z} z=z(1-\tau(k, z)), \quad \bar{\lambda}=\lambda(1+\tau(k, z)), \quad \bar{\Delta} \bar{Z}=\Delta(1+\tau(k, z)),
$$

where

$$
\tau(k, z)=\frac{n_{i} \overline{\vec{A}^{2}}}{4}\left\{\left(4 \cos ^{2} k+\frac{\left(z^{2}-\lambda^{2}\right) \cos 2 k}{J^{2} \Delta^{2}}\right)\left(z^{2}-\lambda^{2}\right)^{-1 / 2}\left(z^{2}-\lambda^{2}+4 J^{2} \Delta^{2}\right)^{-1 / 2}-\frac{\cos 2 k}{J^{2} \Delta^{2}}\right\} .
$$

In this case $\lambda$ and $\Delta$ should also be evaluated consistently. If the impurity density $n_{i}$ is zero, one recovers $\lambda_{0}$ and $\Delta_{0}$. When $n_{i}$ is very small, they can be expanded up to $n_{i}$.

Now we consider the density of the Schwinger boson states for the doped system given by

$$
\frac{d N}{d z}=-\frac{1}{4 \pi} \int \operatorname{Tr} \operatorname{Im} \bar{G}(k, z) \frac{d k}{2 \pi}
$$




$$
\omega_{\text {min }}=\frac{1}{2} E_{H}\left(1-\frac{2}{E_{H}}\left[\frac{\left(\overline{\overrightarrow{A^{2}}}\right)^{2} E_{H}}{4 J^{2} \Delta^{2}}\right]^{1 / 3} n_{i}^{2 / 3}+\frac{\overrightarrow{\overrightarrow{A^{2}}}}{3 J^{2} \Delta^{2}} n_{i}\right),
$$

and $d N / d z$ is zero for $\omega<\omega_{\text {min }}$. Here $E_{H}=2\left(\lambda^{2}-4 J^{2} \Delta^{2}\right)^{1 / 2}$.

In SBMFA, the dynamic structure factor $S(q, \omega)$ is a two-particle Schwinger- boson Green function. The random doping effect consists of two parts. The first is included in the simple product of two single-particle Schwinger-boson Green functions $\bar{G}(k, \omega)$. The second is the correlation effect between two Schwinger bosons induced by scattering at the same impurity. This correlation effect is mostly contributed by the two-particle Green function's ladder diagrams and maximum crossing diagrams. Their contribution is at least proportional to the impurity density $n_{i}$. In the weak doping case we may ignore this effect. Within this approximation $\bar{S}(q, \omega)$ in the presence of impurity scattering can be easily calculated by replacing $G$ in eq. (6) with $\bar{G}$ renormalized by impurity scattering. As in the undoped case, $S(q, \omega)$ vanishes for is the renormalized Haldane gap of the doped sample. If we keep eq.(10) up to the first order of $n_{i}, E_{H}$ and $\Delta$ can be replaced by $E_{H_{0}}$ and $\Delta_{0}$, respectively. Now we make a numerical estimate for the case of non-magnetic doping. Such an experiment has been performed by Glarum et al. [8] For such non-magnetic case we have $\overline{\overrightarrow{A^{2}}}=J_{0}^{2} S(S+1)=8 J^{2}$ (for $S=1$ ). Substituting the parameter values for $\lambda_{0}$ and $\Delta_{0}$ into (10), we find that

$$
\bar{E}_{H}=E_{H_{0}}\left(1-5.6722 n_{i}^{2 / 3}+0.4775 n_{i}\right)
$$

from which we see that $\bar{E}_{H} \approx 0$ when $n_{i} \approx 7.8 \%$. In the SBMFA treatment the VBS state's correlation length $\xi$ is about 10 lattice spacings [12], [13], i.e., almost twice the exact value [4]. Within the same approximation we find that the Haldane gap collapses when the correlation length is comparable with the average impurity distance. This is reasonable and could be expected from our argument at the very beginning.

The doping dependence of the Haldane gap can be observed directly in neutron scattering experiments, for example, in NENP doped with $\mathrm{Cu}$. It will also show up in other physical 
available up to now. However, it has been observed that the spin 1/2 ESR signal decays very rapidly at much lower temperatures than the Haldane gap itself [7], [8]. Mitra, Halperin and Affleck [20] have suggested to explain this effect by considering the thermal excitations which will shift the ESR frequency out of the observation window for short and intermediate chains, while broadening the signal for very long chains. This effect should be there. However, we would suggest that there is a significant reduction of the Haldane gap itself (about $30 \%$ ) even at $1 \%$ doping by non-magnetic impurities due to the effect considered in this Letter. It seems to us that these two complementary aspects should be both taken into account while interpreting the data. Our calculation was done for zero temperature, but it can be easily extended to finite temperatures to compare with actual experiments [18]. It is known [21] that the Haldane gap for undoped systems first incr

In conclusion we have considered the effects of doping by impurity spins in LCHA on the Haldane gap. In this calculation we have considered only the impurity effect on the Schwinger boson continuum spectrum which in turn leads to reduction of the Haldane gap itself, like the early calculation of Gor'kov and Abrikosov [14] for the paramagnetic impurities in superconductors. However, there are bound states in both spin chain problem [9], [12], and superconductors [22]. The bound states in superconductors are rather shallow and it is difficult to observe them experimentally. On the contrary, the bound states in the LCHA are rather deep, very close to the ground state. Hybridizing with the ground state, they give rise to the spin $1 / 2$ signal observed in experiments. They are localized within the correlation length and in the low low doping case we can still focus on the scattering states. On the other hand, if we discuss the collapse of the Haldane gap upon doping quantitatively, both tail states of the continuum band and the band formed by these localized states should be taken into account. In this case the impurity density $n_{i}$ is not very small any longer and we cannot neglect the correlation effect between the Schwinger bosons coming from scattering on the same impurity. In fact, the properties of such gapless LCHA with nonvanishing 
impurity in the normal metal and superconductors. Their quantum fluctuations should have important effects. The work on these issues is in progress and will be reported later.

\section{Acknowledgements}

We would like to thank P. Mitra, T.K. Ng and G.-M. Zhang for helpful discussions. One

of the authors (ZYL) is indebted to Drs. J.-X.Wang, J.-G. Hu and S.-J. Qin for their help. This work was supported in part by the National Natural Science Foundation of China and China Center of Advanced Science and Technology. 


\section{REFERENCES}

[1] F.D.M. Haldane, Phys. Lett. 93A, 464 (1983); Phys. Rev. Lett. 50, 1153 (1983).

[2] I. Affleck, T. Kennedy, E.H. Lieb and H. Tasaki, Phys. Rev. Lett. 59, 799 (1987); Comm. Math. Phys. 115, 477 (1988).

[3] R. Botet \& R. Jullien, Phys. Rev. B27, 613 (1983); M.P. Nightingale \& H.W. Blote, Phys. Rev. B33, 659 (1986).

[4] S. Liang, Phys. Rev. Lett. 64, 1597 (1990).

[5] W. Buyers et al., Phys. Rev. Lett. 56, 371 (1986).

[6] J.P. Renard et al., Europhys. Lett. 3, 945 (1987).

[7] M. Hagiwara et al., Phys. Rev. Lett. 65, 3181 (1990).

[8] S.H. Glarum et al., Phys. Rev. Lett. 67, 1614 (1991).

[9] T.Kennedy, J. Phys.: Cond. Matt. 3, 5757 (1990).

[10] P.W. Anderson, Mater. Res. Bull. 8, 153 (1973)

[11] I. Affleck, Nucl. Phys. B265, 409 (1986); I.Affleck \& F.D.M. Haldane, Phys. Rev. B 36, 5291 (1987).

[12] T.K. Ng, Phys. Rev. B 45, 8181 (1992).

[13] D.P. Arovas \& A. Auerbach, Phys. Rev. B38, 316 (1988).

[14] A.A. Abrikosov \& L.P. Gor'kov, Zh. Exp. Teo. Fis. 39, 1781 (1960).

[15] D.P. Arovas [Phys. Rev. B43, 1255 (1990)] has considered the magnon spectrum in a random $S=1$ LCAF using the "single mode" approximation, but the issue discussed in this Letter, was not addressed there. 
[17] N. Read \& D.M. Newns, J. Phys. C 16, 3273 (1983).

[18] Z.-Y. Lu, Z.-B. Su and L. Yu, to be published.

[19] I. Affleck, J. Phys.: Cond. Mat. 1, 3047, (1989); I. Affleck \& R.A. Weston, Phys. Rev. B 45, 4667 (1992).

[20] P.P. Mitra, B.I. Halperin and I. Affleck, Phys. Rev. B45, 5299 (1992).

[21] See, e.g., P. Gaveau et al., J. Appl. Phys. 69, 5956 (1991)

[22] Lu Yu, Acta Phys. Sinica 21, 75 (1965); H. Shiba, Progr. Theor. Phys. 40, 435 (1968);

A.I. Rusinov, Sov. Phys. JETP Lett. 9, 85 (1969). 